\title{
Hidrografía y plumas estuarinas en Golfo Dulce, Pacífico Sur de Costa Rica
}

\author{
Fernando Rincón-Alejos ${ }^{1} \&$ Daniel Ballestero-Sakson ${ }^{2}$ \\ 1. Programa de Maestría en Ciencias Marinas y Costeras, Universidad Nacional de Costa Rica, Heredia. 86-3000; \\ fernando.rinconalejos@gmail.com \\ 2. Laboratorio de Oceanografía y Manejo Costero, Universidad Nacional de Costa Rica, Heredia. 86-3000; \\ daballest@gmail.com
}

\author{
Recibido 11-VIII-2014. Corregido 01-XII-2014. Aceptado 14-XII-2014.
}

\begin{abstract}
Hidrography and river plumes in Golfo Dulce, south pacific of Costa Rica. Oceanographic variables at 33 stations distributed in Golfo Dulce, Costa Rica, during the dry and rainy seasons between 2009 and 2010, were analyzed with CTD profiles, where salinity, temperature, sigma-T and currents were studied in the water column. Secchi depth and chlorophyll_a were measured in surface. The main objective was to estimate the catchment area and estuarine plume dispersion formed with river discharge. Between the rivers Esquinas and Coto Colorado, decreased salinity $(<30)$ in an area of approximately $250 \mathrm{~km}^{2}$, creating thermohaline fronts along most of the gulf. The El Niño South Oscillation, which was in the phase of heightened was documented. The chlorophyll concentrations correlated $33.7 \%$ with Secchi disc water transparency. The Golfo Dulce surface water was identified with salinity $<31.5$ and temperature $>27^{\circ} \mathrm{C}$ (April to December). This research contributes to the knowledge of the oceanography of the Golfo Dulce and the feedback we receive from fluvial origin, claiming form providing inputs for the potential creation of a major new marine protected area system covering entire Golfo Dulce area and, ideally, the surrounding river systems that feed it. Rev. Biol. Trop. 63 (2): 161-181. Epub 2015 June 01.
\end{abstract}

Key words: Physical oceanography, river discharge, chlorophyll- $a$, El Niño South Oscillation.

Los ríos y drenajes continentales proporcionan la mayor fuente de materiales biogénicos (nitrógeno, fósforo y sílice) a los ecosistemas marino costeros, sosteniendo en muchos casos la producción primaria de las regiones costeras (Mann \& Lazier, 1996; Martínez, Alvarado \& Senior, 2001). En el contexto de bioecosistemas costeros se destacan las zonas estuarinas como ambientes de transición entre océanos y continentes, especialmente aquellos estuarios localizados en los trópicos, que se encuentran entre los más fértiles y productivos (Bonilla, Senior, Bugden, Zafiriou \& Jones, 1993; Secretaría de la Convención Ramsar, 2006). Es acá en donde las plumas estuarinas juegan un papel importante en varios aspectos socio-económicos y ambientales en sus áreas de influencia ya que representan una fuente importante de nutrientes para las aguas costeras, con los consiguientes efectos en la red trófica y en las pesquerías (Mösso et al. 2003).

En la costa Pacífica de Centro América existen condiciones particulares que propician el desarrollo de una gran diversidad biológica. Tal es el caso de Golfo Dulce, al sur de Costa Rica, una zona con características que restringen la circulación del agua transformando sus aguas profundas en un ambiente anóxico, semejante a un fiordo, uno de solo cuatro sistemas conocidos en los trópicos (Richards, 1965; Richards, Anderson \& Cline, 1971; Fairbridge, 1980; Hebbeln, Beese \& Cortés, 1996; Quesada-Alpízar \& Cortés, 2006). Esta zona con pendientes escarpadas, donde convergen condiciones neríticas y oceánicas, mantiene gran parte de su paisaje inalterado prácticamente 
desde su descubrimiento (Acuña-González, Vargas-Zamora \& Córdoba-Muñoz, 2006).

La información actual sobre los procesos de descarga y aporte de nutrientes por parte de los ríos en Golfo Dulce es escasa. Golfo Dulce ha sido identificado como un área de importancia prioritaria para la conservación en el ámbito marino-costero, por el Proyecto GRUAS II Marino-Costero, donde se detallaron los principales objetos de conservación como: fosa anóxica, estuarios, playas de arena gruesa, formaciones coralinas, sitios de alimentación de aves y anidación de tortugas marinas (SINAC-MINAET, 2008). Muchas especies allí observadas encuentran un sitio en donde alimentarse, descansar y aparearse (Silva, 2006), como por ejemplo los delfines nariz de botella Tursiops truncatus, delfines manchados pantropicales Stenella attenuata, ballenas jorobadas Megaptera novaeangliae y falsas orcas Pseudorca crassidens (AcevedoGutiérrez \& Matthew, 2005; Sierra, Vartanián \& Polimeni, 2006; Oviedo, 2008). Aún así, Golfo Dulce posee zonas con diferentes figuras de protección, como $25 \mathrm{~km}^{2}$ dentro del Parque Nacional Piedras Blancas y el Área Marina de Pesca Responsable $\left(773.97 \mathrm{~km}^{2}\right)$.

Actualmente la cuenca de este golfo es objetivo de varios proyectos de desarrollo que pretenden llevarse a cabo en la zona, por lo que para la gestión costera es de particular interés tener las herramientas adecuadas para monitorear y predecir la dinámica de las plumas con el fin de evaluar su influencia a escalas local y regional (Dalsgaard et al., 2003; Mösso et al., 2003; Spongberg, 2004; Ferdelman et al., 2006; Silva \& Acuña-González, 2006).

Los procesos de conservación marina que pretenden proteger o regular el uso y actividades ocurridas en espacios geográficos marinos que no tomen en cuenta el aporte terrestre no se recomiendan. Por esto el objetivo de este estudio es evaluar la hidrografía del Golfo Dulce y estimar el área de influencia de las plumas estuarinas para ser tomado en cuenta en la planificación de estrategias de conservación marina y de la biodiversidad del sistema, conformando una base para el manejo ecosistémico de su cuenca.

\section{MATERIALES Y MÉTODOS}

Área de estudio: Golfo Dulce es un mar interno de origen tectónico, ubicado entre $8^{\circ} 23^{\prime}$ y $8^{\circ} 45^{\prime} \mathrm{N}-83^{\circ} 05^{\prime}$ y $83^{\circ} 30^{\prime} \mathrm{W}$, con una longitud de $50 \mathrm{~km}$ y una anchura entre 10 y $15 \mathrm{~km}$, cubriendo un área aproximada de $680 \mathrm{~km}^{2}$ (Quesada-Alpízar \& Cortés, 2006). Este mar interno se divide en dos sub-áreas importantes: una cuenca interna profunda de pendiente inclinada con un fondo plano de $215 \mathrm{~m}$ de profundidad máxima, y una cuenca externa llana con una profundidad promedio de 70m (Wolff, Hartmann \& Koch, 1996). Un umbral o silla con menos de $30 \mathrm{~m}$ ubicado en su entrada restringe la circulación y hace más lenta la renovación del agua en las capas profundas favoreciendo condiciones anóxicas en el fondo, (Richards et al., 1971).

La marea astronómica, con un rango entre 2 y $4 \mathrm{~m}$ en la parte interna (Svendsen et al., 2006), tiene gran influencia en el litoral y los cursos de agua dulce. Aproximadamente 200ha de manglares están asociadas a los ríos que fluyen al Golfo. El litoral rocoso escarpado con abundancia de bosque tropical domina la línea costera en la zona norte. En el sureste se encuentran playas arenosas como en la Bahía de Pavones y al suroeste desde Playa Platanares, al sur de Puerto Jiménez hasta Cabo Matapalo. La productividad es relativamente baja, con niveles de nutrientes pobres cerca de la superficie, típica de aguas con ausencia de procesos de surgencia.

Se realizaron tres visitas: en enero del 2009 (estación seca), agosto del 2009 (estación lluviosa) y en enero del 2010 (estación seca), con una red de 33 estaciones hidrográficas separadas entre ellas por una distancia entre tres y siete kilómetros aproximadamente (Fig. 1). Se realizaron perfiles verticales de temperatura y salinidad con un CTD (Conductivity, Temperature, and Depth sensor) Falmouth Scientific Inc. 2 Dimensional Acoustic Current Meter (2D ACM) precisión \pm 0.01 . Los 


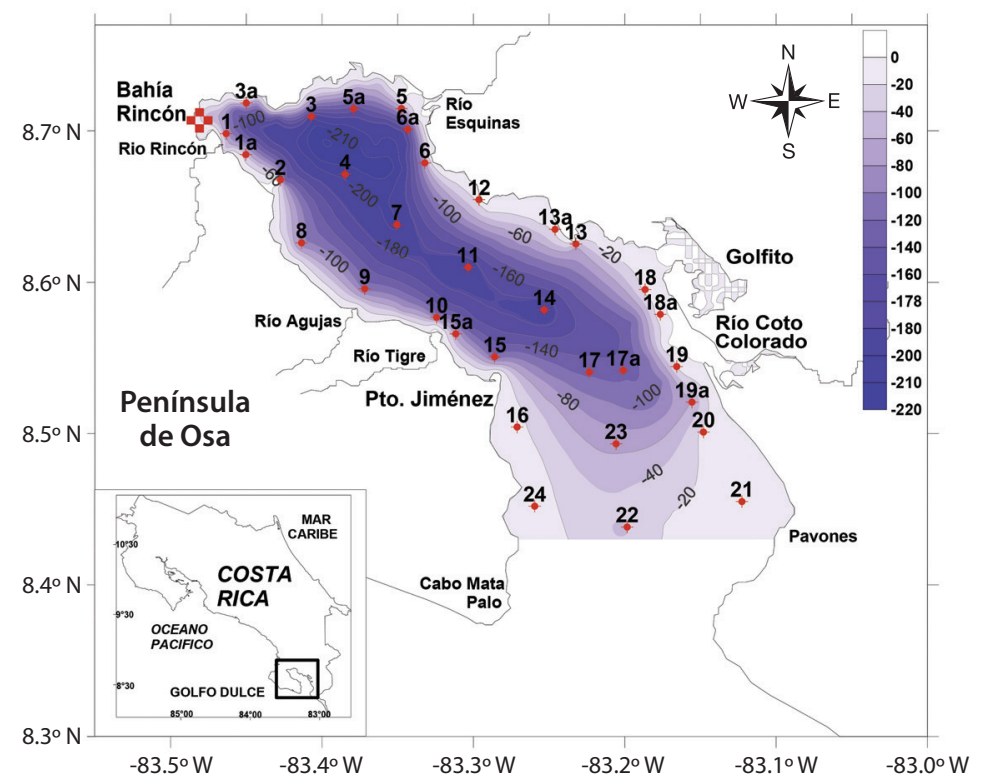

Fig. 1. Ubicación del área de estudio, batimetría (contornos en azul) y estaciones de muestreo (puntos rojos) en Golfo Dulce, Área de Conservación de Osa, Pacífico Sur de Costa Rica. Cruz roja en Bahía Rincón: posición de la estación CM (Ciclo Mareal).

Fig. 1. Study area location, bathymetry (blue contours) and sampling stations (red dots) in Golfo Dulce, Osa Conservation Area, South Pacific of Costa Rica. Red cross in Rincón Bay: CM station position (Tidal Cycle).

datos incluyeron temperatura, salinidad, sigmaT, Disco de Secchi y clorofila- $a$. La salinidad y sigma-T se obtuvieron a partir de la conductividad, usando las fórmulas establecidas por la UNESCO (Fofonoff \& Millard, 1983). Para la determinación de clorofila- $a$ se tomó un litro de agua de mar en superficie y se filtró usando filtros de fibra de vidrio Whatman Glass Microfiber Binder Free, GF/F: $0.7 \mu \mathrm{m}$, se congelaron a $-2^{\circ} \mathrm{C}$ y se analizaron en el Laboratorio de Química Marina de la Universidad Nacional de Costa Rica, antes de transcurrir las primeras 48h. Los filtros fueron tratados con acetona al $90 \%$ y ultrasonido para la extracción de los pigmentos. La concentración de clorofila- $a$ fue determinada por espectrofluorometría siguiendo el método de Holm-Hansen et al. (1965) descrito en Strickland y Parsons (1977).

En enero del 2009 el CTD se llevó a una profundidad máxima de $66 \mathrm{~m}$. En el resto de las visitas el CTD alcanzo mas de los $180 \mathrm{~m}$ de profundidad. Al referirse a un valor promedio en sentido vertical, se incluyen datos únicamente entre 0 y $66 \mathrm{~m}$ de profundidad. La distribución vertical de las variables físicas dentro del golfo fue analizada usando transectos formados con diferentes estaciones de muestreo. La Fig. 2 ilustra los tres diferentes transectos conformados de la siguiente manera: Transecto Central: recorrió longitudinalmente el golfo desde la estación 3 en la cuenca interna hasta la 22 ubicada sobre el umbral; Transecto Coto 1: orientado en sentido este-oeste, desde la estación 19 en la boca del Río Coto Colorado hasta la 15 frente a Puerto Jiménez; Transecto Coto 2: que corta perpendicularmente la boca del Río Coto Colorado.

Para el análisis de la variabilidad estacional de la capa superficial, se escogió para la temperatura la isoterma de $20^{\circ} \mathrm{C}$ por representar el centro de la termoclina según Fiedler y Talley (2006). Al no encontrarse una haloclina definida se escogió para salinidad la isohalina de 34 por su cercanía al límite inferior de 


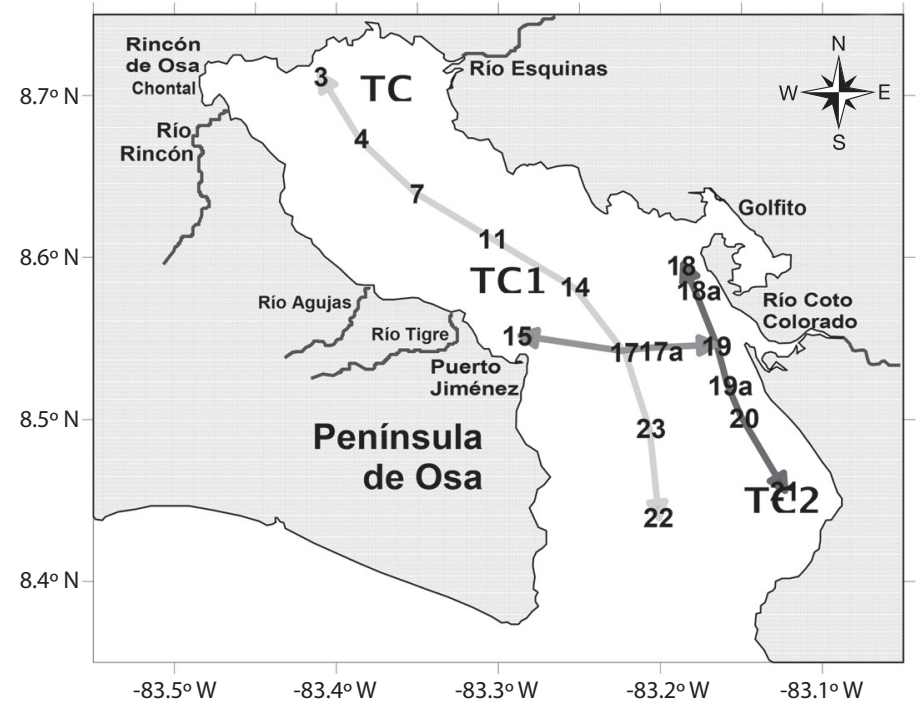

Fig. 2. Transectos donde se analizó la distribución vertical de variables físicas. TC: Transecto Central (de la estación 3 a la 22); TC1: Transecto Coto 1 (de la estación 15 a la 19); TC2: Transecto Coto 2 (de la estación 18 a la 21).

Fig. 2. Transects where the vertical distribution of physical variables was analyzed. TC: Central Transect (from 3 to 22 sample station); TC1: Coto Transect 1 (from 15 to 19 sample station) TC2: Coto Transect 2 (from 18 to 21 sample station).

la capa superficial, usando el mismo criterio para sigma-T donde se escogió la isopicna de $24 \mathrm{~kg} / \mathrm{m}^{3}$.

\section{RESULTADOS}

Temperatura: En enero 2009 (estación seca), la temperatura en superficie (Fig. 3) promedió $29.33 \pm 0.49^{\circ} \mathrm{C}$ (Cuadro 1), con un máximo de $30.51^{\circ} \mathrm{C}$ en la estación $3 \mathrm{a}$ y un mínimo de 27.98 en la estación 16. En general, la totalidad de los datos arrojó una media de $25.18 \pm 5.29^{\circ} \mathrm{C}$. En agosto 2009 (estación lluviosa), los valores en superficie estuvieron entre $30.57^{\circ} \mathrm{C}$ en la estación 18 , y $28.72^{\circ} \mathrm{C}$ en la estación 4, con una media $29.80 \pm 0.36^{\circ} \mathrm{C}$. El promedio general dentro del golfo fue $22.15 \pm 6.19^{\circ} \mathrm{C}$ durante la estación lluviosa. Para la estación seca en enero 2010 los valores fueron en promedio más altos con respecto a las giras anteriores, con una media en superficie (Cuadro 1) de $30.67 \pm 0.46^{\circ} \mathrm{C}$ y general de $22.98 \pm 6.16^{\circ} \mathrm{C}$. En superficie, el valor máximo fue $32.03^{\circ} \mathrm{C}$ en la estación $13 \mathrm{a}$, mientras que el mínimo fue $29.92^{\circ} \mathrm{C}$ en la estación 24 .
La Fig. 4 muestra la distribución vertical de temperatura a lo largo del Transecto Central (TC) en Golfo Dulce. Durante enero del 2009 puede observarse la termoclina entre los $20 \mathrm{y}$ los $60 \mathrm{~m}$, con valores de $29^{\circ} \mathrm{C}$ en superficie y $17^{\circ} \mathrm{C}$ cerca de los $60 \mathrm{~m}$ de profundidad. Nótese que no hay datos por debajo de los $66 \mathrm{~m}$ en enero 2010. La Fig. 5 muestra la isoterma de $20^{\circ} \mathrm{C}$ para las tres visitas realizadas, localizada entre los 40 y 50m durante enero 2009. El gradiente desde la superficie hasta los $66 \mathrm{~m}$ fue de $12^{\circ} \mathrm{C}$. Durante agosto 2009 , la temperatura en el TC mostró un gradiente entre la superficie y los $66 \mathrm{~m}$ de $13^{\circ} \mathrm{C}$, con valores desde $30^{\circ} \mathrm{C}$ a $0 \mathrm{~m}$ hasta poco menos de $17^{\circ} \mathrm{C}$ a los $66 \mathrm{~m}$ en la estación 11. Durante esta estación lluviosa la termoclina se ubicó entre los 35 y $60 \mathrm{~m}$ de profundidad. En la Fig. 5 puede verse la isoterma de $20^{\circ} \mathrm{C}$ entre los 50 y $60 \mathrm{~m}$ en la cuenca interna, ascendiendo hasta $45-55 \mathrm{~m}$ en la cuenca externa. En enero 2010 la termoclina volvió a ubicarse entre los 30 y $60 \mathrm{~m}$ de profundidad (Fig. 4). El gradiente hasta los $66 \mathrm{~m}$ fue de $12^{\circ} \mathrm{C}$, con valores de $30^{\circ} \mathrm{C}$ en superfície y $18.06^{\circ} \mathrm{C}$ a $65.7 \mathrm{~m}$ en la estación 10 . La isoterma 

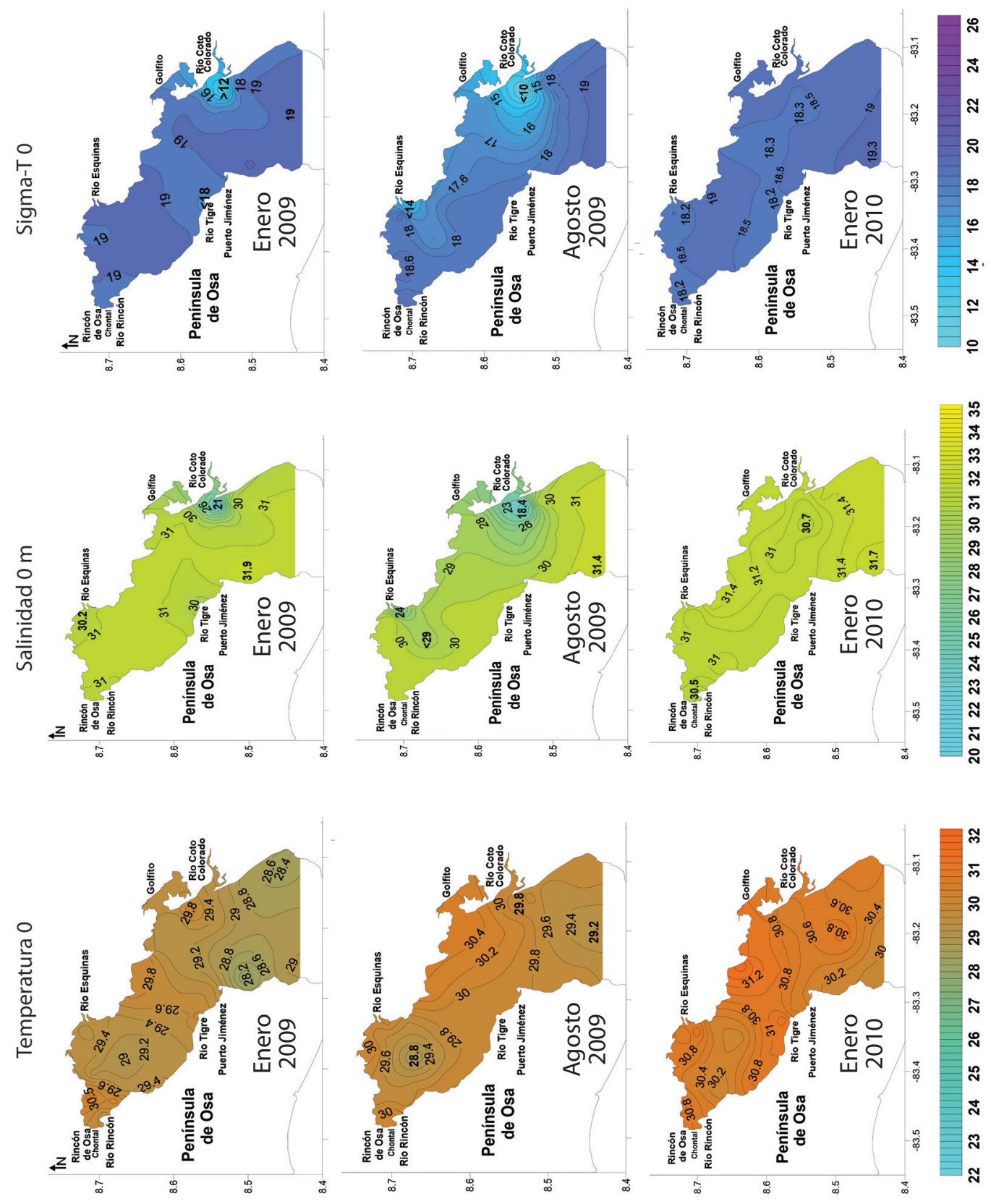

Fig. 3. Distribución de temperatura $\left({ }^{\circ} \mathrm{C}\right.$, columna izq.), salinidad (columna central) y sigma-T (kg.m ${ }^{3}$, columna der.) en superficie durante enero 2009 (arriba), agosto 2009 (centro) y enero 2010 (abajo).

Fig. 3. Distribution of temperature $\left({ }^{\circ} \mathrm{C}\right.$, left column), salinity (middle column) and sigma-T (kg.m ${ }^{3}$, right column) at the surface during january 2009 (above), august 2009 (center) and january 2010 (bottom). 
CUADRO 1

Media, desviación estándar, mínimo y máximo de las variables estudiadas en Golfo Dulce para todas las visitas a nivel superficial

TABLE 1

Mean average, standard deviation, minimum and maximum of variables studied in Golfo Dulce for each survey in the surface

\begin{tabular}{|c|c|c|c|c|c|}
\hline & Salinidad & Temperatura $\left({ }^{\circ} \mathrm{C}\right)$ & Sigma- $\mathrm{T}\left(\mathrm{Kg} / \mathrm{m}^{3}\right)$ & Disco de Secchi (m) & Clorofila- $a(\mathrm{uMol} / \mathrm{L})$ \\
\hline \multicolumn{6}{|c|}{ Estación seca, enero 2009} \\
\hline Media: & 30.64 & 29,33 & 18.71 & 10.27 & 23.66 \\
\hline$D S:$ & 1.84 & 0.49 & 1.40 & 4.85 & 26.58 \\
\hline Min: & 20.82 & 27.98 & 11.39 & 0.50 & 1.34 \\
\hline Máx: & 31.99 & 30.51 & 20.14 & 18.00 & 91.67 \\
\hline \multicolumn{6}{|c|}{ Estación lluviosa, agosto 2009} \\
\hline Media: & 29.29 & 29.80 & 17.53 & 4.53 & 2.64 \\
\hline$D S:$ & 2.71 & 0.36 & 2.04 & 2.80 & 1.34 \\
\hline Min: & 18.37 & 28.72 & 9.42 & 0.40 & 0.90 \\
\hline Máx: & 31.42 & 30.57 & 19.16 & 9.60 & 5.16 \\
\hline \multicolumn{6}{|c|}{ Estación seca, enero 2010} \\
\hline Media: & 31.11 & 30.66 & 18.59 & 10.37 & 0.46 \\
\hline$D S:$ & 0.29 & 0.46 & 0.29 & 5.38 & 0.40 \\
\hline Min: & 30.54 & 29.92 & 18.14 & 0.80 & 0.02 \\
\hline Máx: & 31.77 & 32.03 & 19.34 & 21.50 & 1.32 \\
\hline
\end{tabular}

de $20^{\circ} \mathrm{C}$ se encontró entre los 50 y $60 \mathrm{~m}$ de profundidad (Fig. 5) durante la estación lluviosa.

La estructura vertical de temperatura muestra una capa superficial bastante homogénea, de poco menos de $20 \mathrm{~m}$ de grosor y $29^{\circ} \mathrm{C}$ en la estación lluviosa, más somera y menos definida en la estación seca (enero 2009) en la parte interna del GD (Fig. 4), observándose una termoclina localizada entre 30 y $50 \mathrm{~m}$ en enero 2009 y entre 30 y $60 \mathrm{~m}$ en agosto 2009 . La estructura térmica es notablemente distinta en enero 2010 durante ENOS, donde la estratificación se extiende hasta la superficie y la termoclina se localiza entre 40 y $10 \mathrm{~m}$ de profundidad. La elevación de la termoclina en enero 2010 fue consistente con la disminución del grosor de la capa superficial, resultante del escaso aporte de agua dulce evidenciado en la Fig. 4.

Salinidad: La distribución de la salinidad superficial en cada campaña (Cuadro 1) puede verse en la Fig. 3. Para la primera visita en enero del 2009, la salinidad en superficie promedió $30.64 \pm 1.83$ con valores entre 20.82 en la estación 19 frente al Río Coto Colorado y 31.99 en estación 16 cercana a Puerto Jiménez.
En sentido vertical se obtuvo un promedio de $32.46 \pm 1.95$ más baja en comparación con la siguiente estación seca (enero 2010). Durante la estación lluviosa de 2009, la salinidad fue más baja en los primeros metros de la columna de agua, con una media en superficie de $29.29 \pm 2.71$. El menor valor fue 18.37 en la estación 19 y un máximo de 31.42 en la estación 24 para datos en superficie. La mayor salinidad obtenida a profundidad para la temporada lluviosa fue 34.99 encontrada por debajo de los $175 \mathrm{~m}$ en la estación 4 y la mínima fue 18.37. La media general fue $33.92 \pm 1.74$, más alta que en enero del 2010, donde la media general fue $33.35 \pm 1.77$ con un máximo de 34.96 en la estación 10 a $65.7 \mathrm{~m}$ y un mínimo de 19.49 en la estación 19 a $11 \mathrm{~m}$, donde se notó la presencia de agua más dulce entre 11 y $12 \mathrm{~m}$, por debajo de agua más salada, es decir, valores de 30.80 en superficie sobre valores de 19 y 20 a $11 \mathrm{~m}$. Por esta singularidad en los datos, se excluyó la estación 19 del análisis para la media general de enero 2010. La media para los datos en superficie durante enero 2010 resultó $31.11 \pm 0.29$. Durante esta última visita realizada (enero 2010) no se registró a 

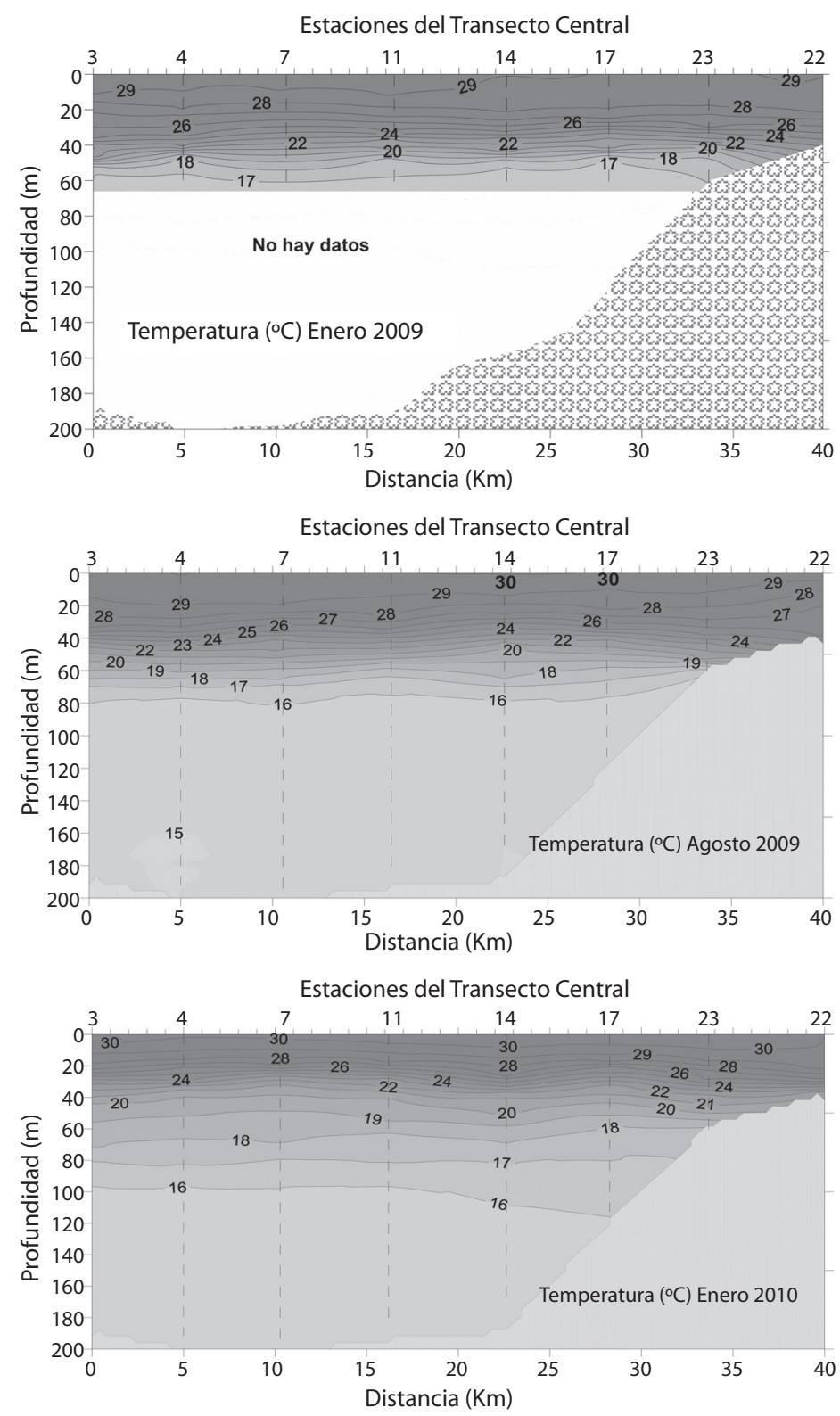

Fig. 4. Distribución vertical de temperatura $\left({ }^{\circ} \mathrm{C}\right.$ ) en el Transecto Central durante enero 2009 (arriba), agosto 2009 (centro) y enero 2010 (abajo).

Fig. 4. Temperature vertical distribution $\left({ }^{\circ} \mathrm{C}\right)$ in the Center Transect during January 2009 (top), August 2009 (center) and January 2010 (bottom).

nivel superficial y en toda el área de muestreo, salinidad por debajo de 30.54 ni por encima de 31.77 .

La Fig. 6 muestra los valores de salinidad a lo largo del transecto TC para todas las campañas. Durante enero 2009 puede observarse una estratificación salina superficial, con la isohalina de 34 rondando los $40 \mathrm{~m}$ en la cuenca interna y casi los $50 \mathrm{~m}$ en la cuenca externa (Fig. 5). En agosto 2009 se muestra la misma 

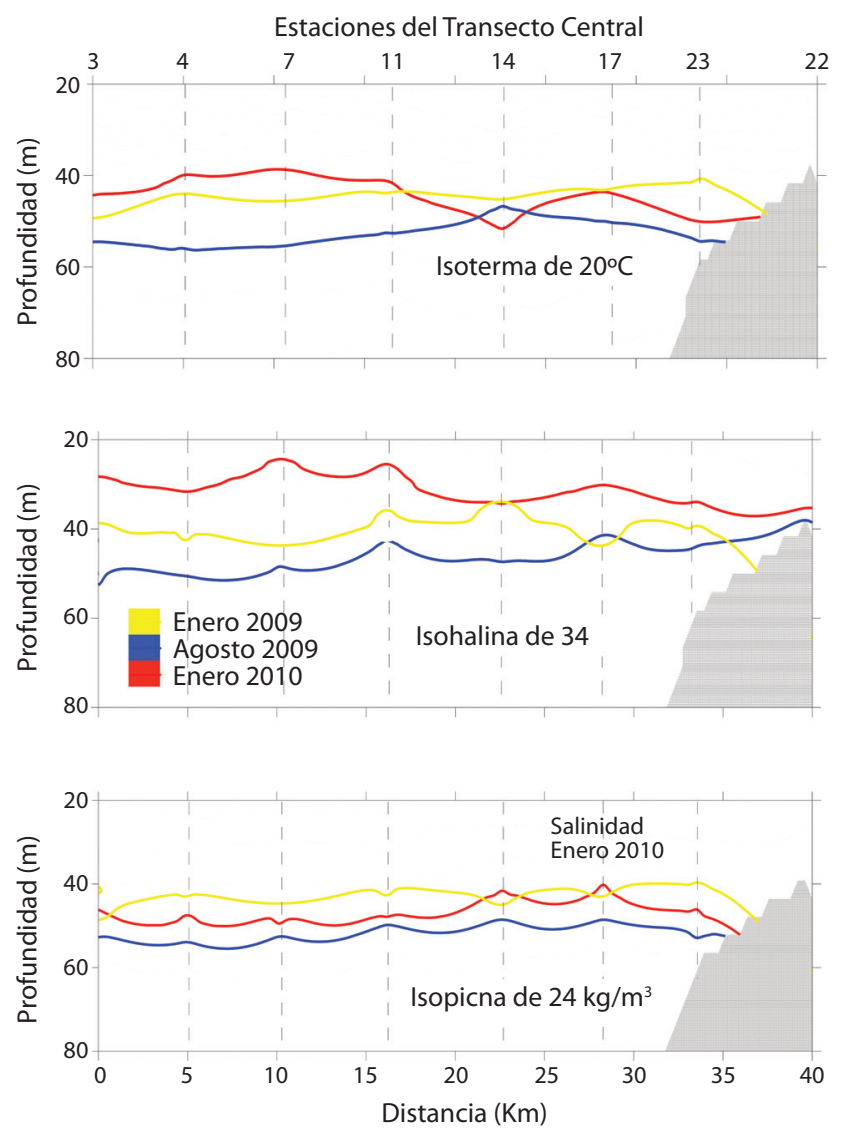

Fig. 5. Isoterma de $20^{\circ} \mathrm{C}$ (arriba), Isohalina de 34 (centro) e Isopicna de $24 \mathrm{~kg} / \mathrm{m}^{3}$ (abajo) durante enero 2009 (amarillo), agosto 2009 (azul) y enero 2010 (rojo).

Fig. 5. $20^{\circ} \mathrm{C}$ isotherm (above), isohaline of 34 (middle) and $24 \mathrm{~kg} / \mathrm{m}^{3}$ isopycnal (bottom) during January 2009 (yellow), August 2009 (blue) and January 2010 (red).

estratificación pero más cercana a la superficie, con valores $<27$ a $0 \mathrm{~m}$ en la estación 17 (Fig. 6) y la isolhalina de 34 ubicada cerca de los $50 \mathrm{~m}$ en la cuenca interna y $40 \mathrm{~m}$ en la cuenca externa, al contrario que en la estación seca del mismo año. Los valores de salinidad para enero 2010 ubican la isohalina de 34 entre los 20 y $30 \mathrm{~m}$ de profundidad en la cuenca interna y entre los 30 y $40 \mathrm{~m}$ en la cuenca externa, con valores mínimos de 31 en superficie. En todos los casos se observa estratificación salina desde la superficie sin una haloclina definida.

Sigma-T $\left(\sigma_{t}=\rho-1000\right):$ La distribución horizontal de sigma-T en superficie durante la estación seca 2009 (Fig. 3) varió entre $11.39 \mathrm{~kg} /$ $\mathrm{m}^{3}$ en la estación 19 y $25.8 \mathrm{~kg} / \mathrm{m}^{3}$ en la estación 11, con una media a nivel superficial de $18.71 \pm 1.40 \mathrm{~kg} / \mathrm{m}^{3}$ (Cuadro 1). La mayor concentración para esta visista (enero 2009) fue $\sigma_{\mathrm{t}}=25.79 \mathrm{~kg} / \mathrm{m}^{3}$ localizado a $\operatorname{los} 65 \mathrm{~m}$ de profundidad en las estaciones 9 y 10 , con un promedio general de $21.42 \pm 2.76 \mathrm{~kg} / \mathrm{m}^{3}$. La Fig. 7 muestra la distribución vertical de sigma-T para todas las visitas. En enero 2009 el mayor gradiente estuvo entre los 15 y los $60 \mathrm{~m}$, con valores por debajo de $19 \mathrm{~kg} / \mathrm{m}^{3}$ en superficie y $>25 \mathrm{~kg} / \mathrm{m}^{3}$ a los $60 \mathrm{~m}$. La isopicna de $24 \mathrm{~kg} / \mathrm{m}^{3}$ se ubicó entre los 40 y $50 \mathrm{~m}$ de profundidad en ambas cuencas del golfo (Fig. 5). 

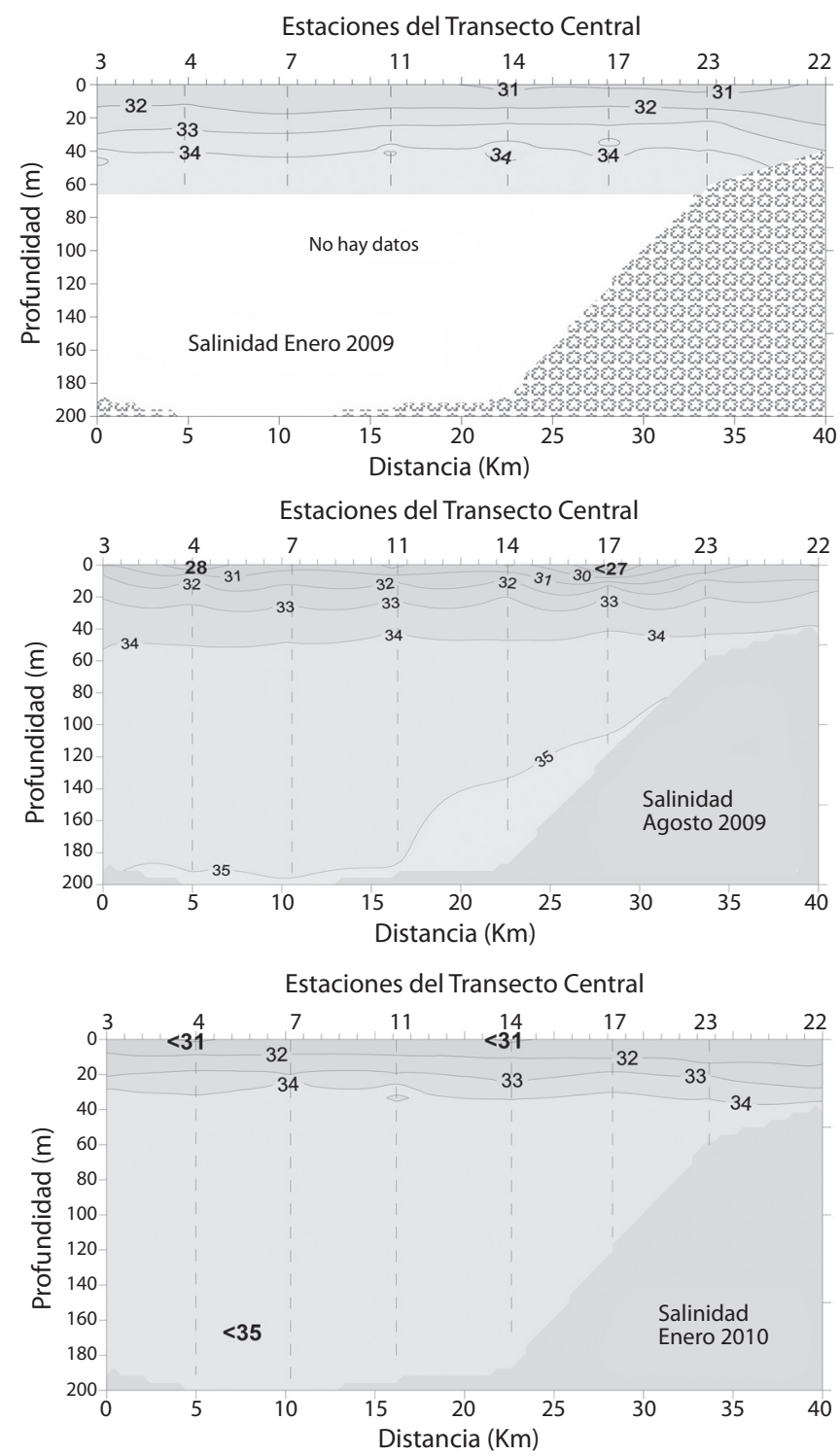

Fig. 6. Distribución vertical de salinidad en el Transecto Central durante enero 2009 (arriba), agosto 2009 (centro) y enero 2010 (abajo).

Fig. 6. Salinity vertical distribution in the Central Transect during January 2009 (top), August 2009 (center) and January 2010 (bottom).

Durante la estación lluviosa del 2009 sigma-T en superficie (Fig. 3) promedió $17.53 \pm 2.04 \mathrm{~kg} / \mathrm{m}^{3}$ (Cuadro 1). El mínimo valor fue $9.42 \mathrm{~kg} / \mathrm{m}^{3}$ ubicado a $0 \mathrm{~m}$ en la estación 19 , coincidiendo con la salinidad más baja (18.37 a $0 \mathrm{~m}$ ). El valor máximo durante esta visita fue de $26.79 \mathrm{~kg} / \mathrm{m}^{3}$, hallado en las estaciones $3 \mathrm{y}$ 4 a $186 \mathrm{~m}$, la máxima profundidad alcanzada por el CTD durante la estación lluviosa 2009 (187.2m). El valor promedio fue $21.41 \pm 2.53 \mathrm{~kg} /$ $\mathrm{m}^{3}$. La estación lluviosa del 2009 mostró un gradiente mayor entre la superficie y los $66 \mathrm{~m}$ 

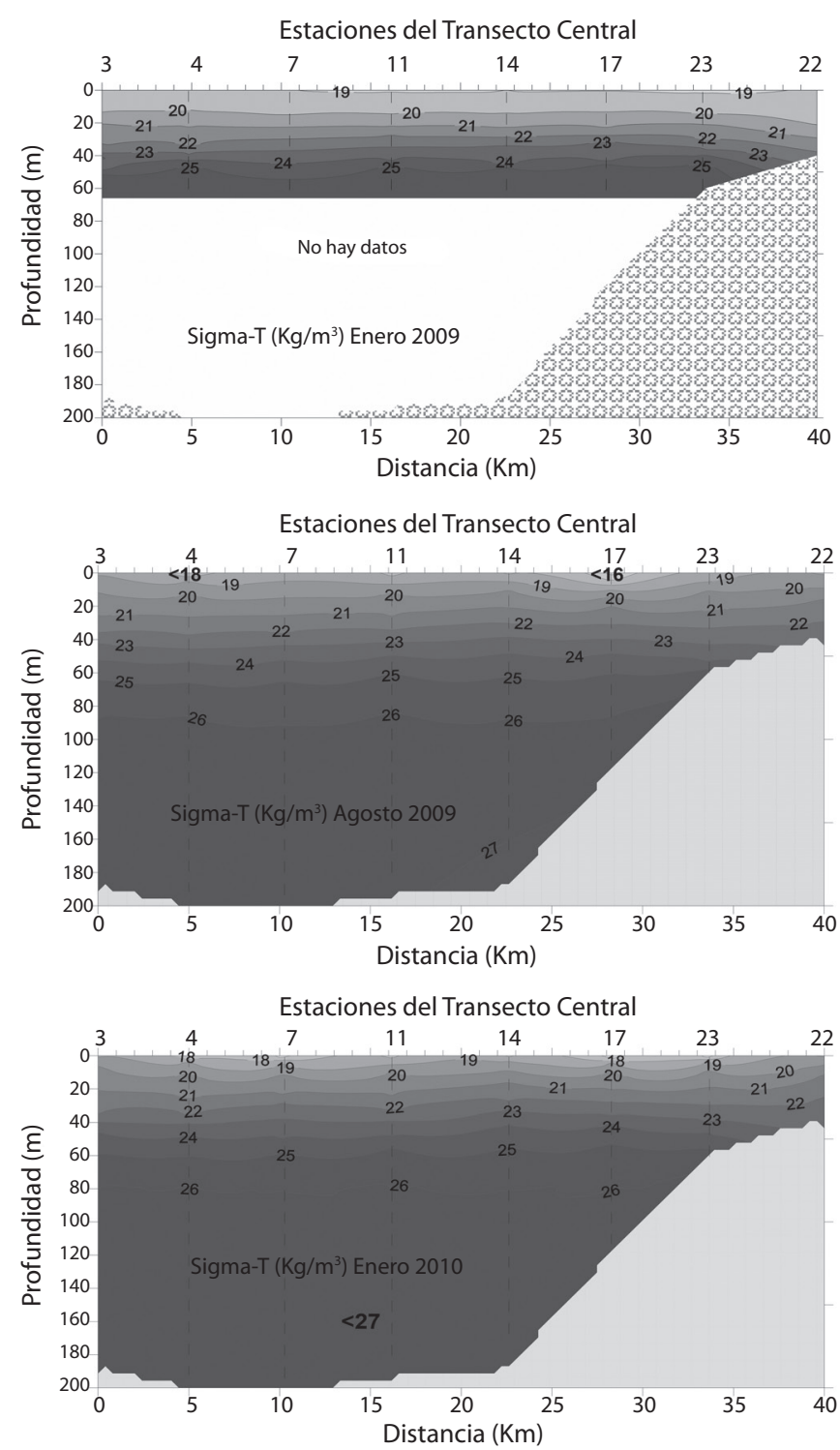

Fig. 7. Distribución vertical de sigma-T $\left(\mathrm{kg} / \mathrm{m}^{3}\right.$ ) en Transecto Central durante enero 2009 (arriba), agosto 2009 (centro) y enero 2010 (abajo).

Fig. 7. Sigma-T vertical distribution $\left(\mathrm{kg} / \mathrm{m}^{3}\right)$ in Central Transect during January 2009 (top), August 2009 (center) and January 2010 (bottom).

de profundidad, con un valor $<16 \mathrm{~kg} / \mathrm{m}^{3}$ en la estación 17 y valores $>25 \mathrm{~kg} / \mathrm{m}^{3}$ por debajo de los $60 \mathrm{~m}$ en algunas estaciones de la cuenca externa (Fig. 7). La isopicna de $24 \mathrm{~kg} / \mathrm{m}^{3}$ (Fig. 5) se localiza ligeramente por debajo de los $50 \mathrm{~m}$ de profundidad en la cuenca interna y muy levemente por encima en la cuenca externa.
Cabe mencionar que la densidad dentro de Golfo Dulce está determinada prácticamente por la salinidad.

En enero 2010 la superficie mostró una media de $18.59 \pm 0.29 \mathrm{~kg} / \mathrm{m}^{3}$ (Cuadro 1) dentro de un rango que va de $18.14 \mathrm{~kg} / \mathrm{m}^{3}$ en la estación $6 \mathrm{a}$, hasta $19.34 \mathrm{~kg} / \mathrm{m}^{3}$ en la estación 24 (Fig. 3). 
La media general fue $21.33 \pm 2.62 \mathrm{~kg} / \mathrm{m}^{3}$. El agua menos densa se ubicó a los $11.1 \mathrm{~m}$ de profundidad en la estación 19 , con $\sigma_{\mathrm{t}}=10.71 \mathrm{~kg} /$ $\mathrm{m}^{3}$. Dicha estación se excluyó del análisis por presentar comportamiento atípico. En esta estación seca se alcanzó una profundidad con el CTD de $193.5 \mathrm{~m}$ en la estación 3, donde se registró la mayor densidad $\sigma_{\mathrm{t}}=26.74 \mathrm{~kg} / \mathrm{m}^{3}$. Se observó en la distribución vertical de sigma-T (Fig. 7) estratificación en la columna de agua desde la superficie hacia el fondo, acentuada en los primeros $50 \mathrm{~m}$, más que una picnoclina realmente establecida o bien marcada. La Fig. 5 muestra la profundidad de la isopicna de $24 \mathrm{~kg} /$ $\mathrm{m}^{3}$ durante enero 2010; esta se ubica alrededor de 1 los $50 \mathrm{~m}$ en la cuenca interna y sube hasta casi los $40 \mathrm{~m}$ en la cuenca externa.

Los resultados muestran claramente el notable impacto que tiene el aporte de agua fresca sobre la hidrografía del cuerpo de agua del GD. La variabilidad estacional de la estructura vertical de temperatura y salinidad de la capa superior ha sido documentada entre las estaciones seca (enero) y lluviosa (agosto) del año 2009, en tanto que los datos obtenidos en enero 2010, durante uno de los eventos de El Niño-Oscilación del Sur (ENOS) más intensos de los últimos años, documentan las condiciones extremas que puede alcanzar el GD en días de muy baja precipitación y alta insolación. $\mathrm{La}$ variabilidad estacional e interanual de la capa superior del GD es ilustrada a lo largo del transecto central (TC, Fig. 5), que integra espacial y temporalmente los efectos del intercambio de calor y agua fresca del GD. Los cambios en la capa superior son ilustrados por la profundidad de la isoterma de $20^{\circ} \mathrm{C}$, que en GD se localiza en la parte inferior de la termoclina local, y por la isohalina de 34 , que se ubica en la interfase entre la capa superior afectada por el intercambio de agua fresca con la atmósfera y el continente, y la capa profunda aislada del forzamiento externo.

La variabilidad estacional e interanual tuvo máxima amplitud en la parte interna del GD (estaciones 3 a 11, Fig. 5), particularmente en la salinidad, en tanto que en la parte externa (estaciones 17 a 22, Fig. 5) las isolíneas tendieron a converger hacia las condiciones más estables del ambiente oceánico, con evidencia de forzamiento topográfico por la silla a la entrada del GD sobre la limitada circulación estuarina e intercambio de agua GD-Océano Pacífico. Tomando la isohalina de 34 como la base de la capa superior forzada por las condiciones meteorológicas, se observó un ensanchamiento de la capa superior, en la parte interna del golfo, del orden de $10 \mathrm{~m}$ entre la estación seca y la estación lluviosa (desde 40 a $50 \mathrm{~m}$ de profundidad entre enero y agosto de 2009), pero esta diferencia en el grosor de la capa superior aumentó a $25 \mathrm{~m}$ (de 50 a $25 \mathrm{~m}$ de profundidad entre agosto 2009 y enero 2010) si consideramos las condiciones de escasa precipitación durante ENOS 2010.

La variabilidad en la profundidad de la isoterma de $20^{\circ} \mathrm{C}\left(\mathrm{h}_{20}\right)$, tomada como una medida del grosor de la capa superficial y la parte superior de la termoclina en la parte interna del GD, mostró un comportamiento similar pero de menor amplitud al de la isohalina de 34 . La diferencia en $h_{20}$ entre enero 2010 y agosto 2009 es de $17 \mathrm{~m}$ (hundimiento desde 40 a 57m), pero la diferencia máxima entre las profundidades de $\mathrm{h}_{20}$ entre enero 2009 y enero 2010 es de sólo $5 \mathrm{~m}$, evidenciando que, a diferencia de la salinidad, la variabilidad estacional en $\mathrm{h}_{20}$ es mayor que la variabilidad interanual.

Los valores de salinidad y sigma- $T$ en superficie (Fig. 2) ilustran cómo se dispersó el agua dulce que entró al golfo a través de los ríos Esquinas y Coto Colorado. En enero 2009 únicamente se distinguió a nivel superficial la presencia del Río Coto Colorado dispersando su pluma desde su desembocadura hacia el oeste sobre un área $>30 \mathrm{~km}^{2}$, mientras que en agosto 2009 se observaron las plumas del Río Coto Colorado y la del Esquinas con aguas menos densas (sigma- $\mathrm{T} \leq 18 \mathrm{~kg} / \mathrm{m}^{3}$ ) en la zona de sus respectivas desembocaduras. Entre ambos ríos ocuparon un área aproximada de unos $250 \mathrm{~km}^{2}$, cubriendo casi la totalidad de la costa este del Golfo Dulce y delineando un gran frente termohalino que abarcó tanto la cuenca interna como la externa del golfo. Agua más densa en superficie (sigma-T $>19 \mathrm{~kg} / \mathrm{m}^{3}$ ) se 
Salinidad

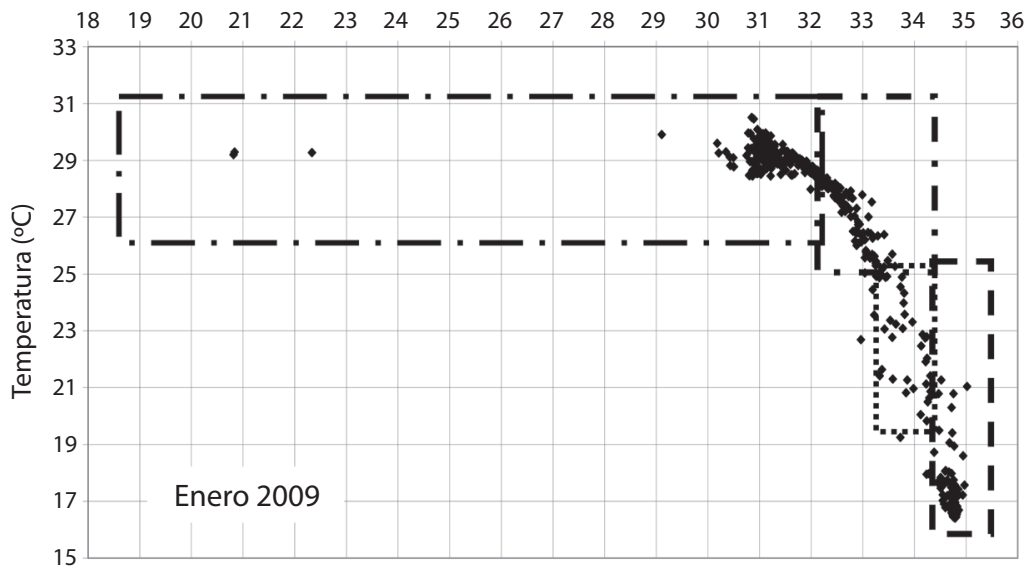

Salinidad

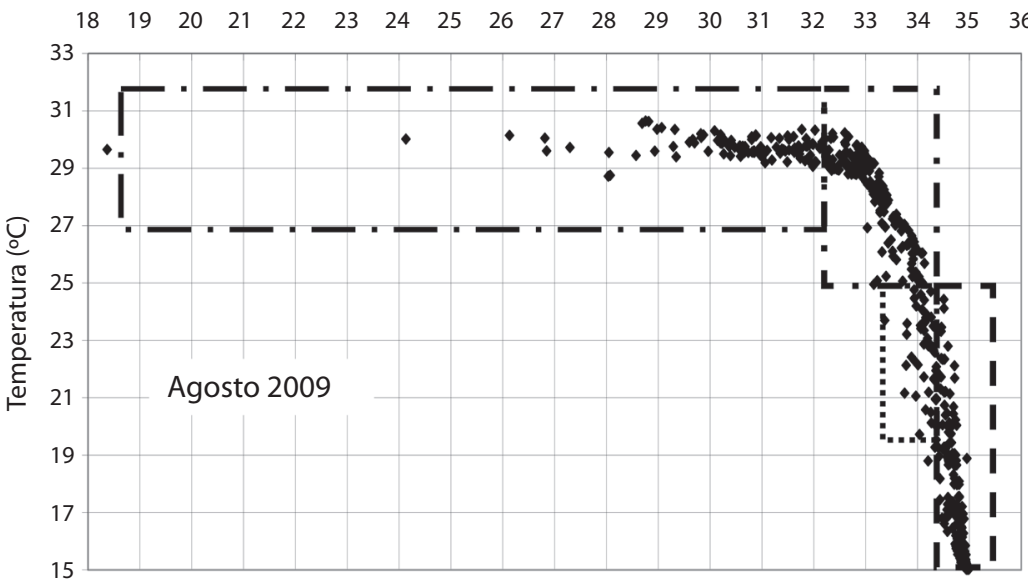

Salinidad

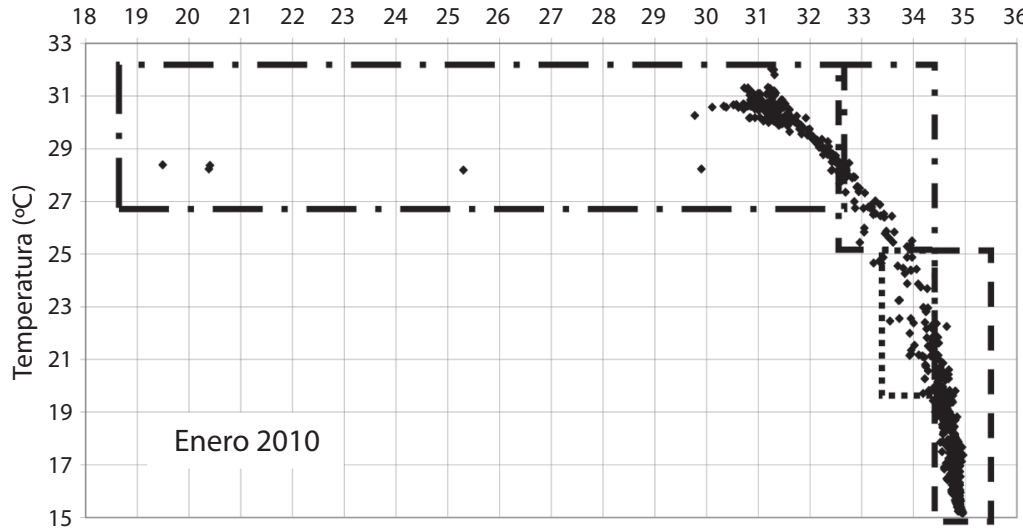

Fig. 8. Diagramas TS. Rectángulo líneas y puntos: Agua Tropical Superficial (ATS); lineas: Agua Ecuatorial Superficial (AES); líneas largas y puntos: capa superficial del Golfo Dulce; puntos: mezcla entre ATS y AES.

Fig. 8. TS diagrams for each survey. Dash dot rectangle: Tropical Surface Water (TSW); dash: Equatorial Surface Water (ESW); long dash dot: surface layer of the Golfo Dulce; dot: TSW and AES mixing. 
observa entrando al golfo desde mar abierto por el sureste, detectada en las estaciones 22 y 24.

Diagramas T-S: Los datos de temperatura y salinidad identifican las masas de agua presentes en Golfo Dulce (Fig. 8). Se observan claramente la masa de Agua Ecuatorial Superficial (AES), la masa de Agua Tropical Superficial (ATS) y una cantidad de datos con valores de temperatura entre 19 y $25^{\circ} \mathrm{C}$ y de salinidad entre 33 y 34 que probablemente se deba a la mezcla entre ambas. Dentro del rectángulo con líneas largas y puntos se encuentra la capa superficial del Golfo Dulce, con salinidad $<31.5$ y temperatura $>27^{\circ} \mathrm{C}$, localizada en los primeros cinco metros de la columna de agua, ilustrando la gran influencia de origen continental.

Disco Secchi: La transparencia del agua según la lectura del disco Secchi durante la estación seca en enero 2009, promedió $10.27 \pm 4.85 \mathrm{~m}$ (Cuadro 1), con un mínimo de $0.5 \mathrm{~m}$ en la estación 19 ubicada frente a la boca del Río Coto Colorado, y un máximo de $18 \mathrm{~m}$ en las estaciones 6 y 11 (Fig. 9). Esta variable para la estación lluviosa reveló las aguas menos transparentes, obteniendo una media $4.53 \pm 2.80 \mathrm{~m}$ (Cuadro 1), con valores entre los $0.4 \mathrm{~m}$ en la estación 5 frente al Río Esquinas y como valor máximo $9.6 \mathrm{~m}$ en la 9 cerca de la boca del Río Agujas. En enero 2010 la capa superficial dentro del Golfo mostró mayor transparencia y la visibilidad alcanzó una profundidad de $21.5 \mathrm{~m}$ en la estación 3 como el valor más alto obtenido en todas las campañas. El mínimo durante esta última campaña fue $0.8 \mathrm{~m}$ en la $17 \mathrm{a}$. La media fue $10.37 \pm 5.38 \mathrm{~m}$ (Cuadro 1).

Clorofila- $\boldsymbol{a}$ : Los valores de Clorofila- $a$ obtenidos durante enero del 2009 resultaron ser los más altos. Nótese que en la escala de valores del contorno de clorofila- $a$ (Fig. 9), el máximo es $6.5 \mu \mathrm{Mol} / \mathrm{L}$. En enero 2009 se registraron los valores más altos, muy por encima de dicha escala, con una media $23.66 \pm 26.58 \mu \mathrm{Mol} / \mathrm{L}$ (Cuadro 1), entre un mínimo de 1.34 en la estación 3 y un máximo de $91.67 \mu \mathrm{Mol} / \mathrm{L}$ en la estación 18 frente a la boca de Golfito. Durante la estación lluviosa 2009, los valores estuvieron entre $0.897 \mu \mathrm{Mol} / \mathrm{L}$ en las estaciones 1,2 y 22 y $5.16 \mu \mathrm{Mol} / \mathrm{L}$ en las estaciones 16 y 20. La media fue $2.64 \pm 1.34 \mu \mathrm{Mol} / \mathrm{L}$ (Cuadro 1). Para enero del 2010 (Cuadro 1) los valores disminuyeron, obteniéndose los más bajos de las tres visitas realizadas, con promedio $0.46 \pm 0.40 \mu \mathrm{Mol} / \mathrm{L}$ de $\mathrm{Cl} \_a$, desde concentraciones ND en 14 de las 24 estaciones visitadas, pasando por valores de $0.022 \mu \mathrm{Mol} / \mathrm{L}$ en la estación 22 , hasta un máximo de $1.32 \mu \mathrm{Mol} / \mathrm{L}$ en la estación 20.

Entre junio y agosto 2008 se realizó un muestreo preliminar donde se tomaron muestras para analizar clorofila- $a$ obteniendo una media de $\mathrm{Cl} a=6.9 \pm 15.0 \mu \mathrm{Mol} / \mathrm{L}$, un máximo de $88.8 \mu \mathrm{Mol} / \mathrm{L}$ en la estación 17 y valores de $11.45 \mu \mathrm{Mol} / \mathrm{L}$ en la estación 5 frente al Río Esquinas, $8 \mu \mathrm{Mol} / \mathrm{L}$ en la estación 15 frente al Río Tigre y $5.9 \mu \mathrm{Mol} / \mathrm{L}$ en la $3 a$, una de las más cercana a la boca del Río Rincón. En dicha ocasión se tomaron fotografías (no se muestran) donde se observa una posible floración algal extraordinaria.

Se encontró relación significativa entre la concentración de clorofila- $a$ y la transparencia del agua según la profundidad del disco de Secchi para los datos correspondientes a la estación lluviosa, arrojando un $\mathrm{R}^{2}=0.337$ $(\mathrm{P}=0.05)$ como se observa en la Fig. 14. En esta temporada los máximos de clorofila se observaron cerca de los frentes que se forman en Golfo Dulce según las figuras producidas a partir de las medidas de la transparencia del agua con el disco de Secchi (Fig. 9)

La Fig. 10 muestra el modelo del perfil de salinidad obtenido en este estudio, mientras que la Fig. 11 grafica la distribución vertical de salinidad en el transecto TC2. La Fig. 12 muestra la distribución vertical de sigma-T a lo largo del transecto TC1 (Fig. 3). Claramente se observa cómo la pluma del Río Coto Colorado durante 2009, se dispersa desde la estación 19 hacia el oeste (estaciones 17a y 17) y ocupa los primeros metros de la columna de agua. El grosor de esta pluma (4.66m en 2009) va 


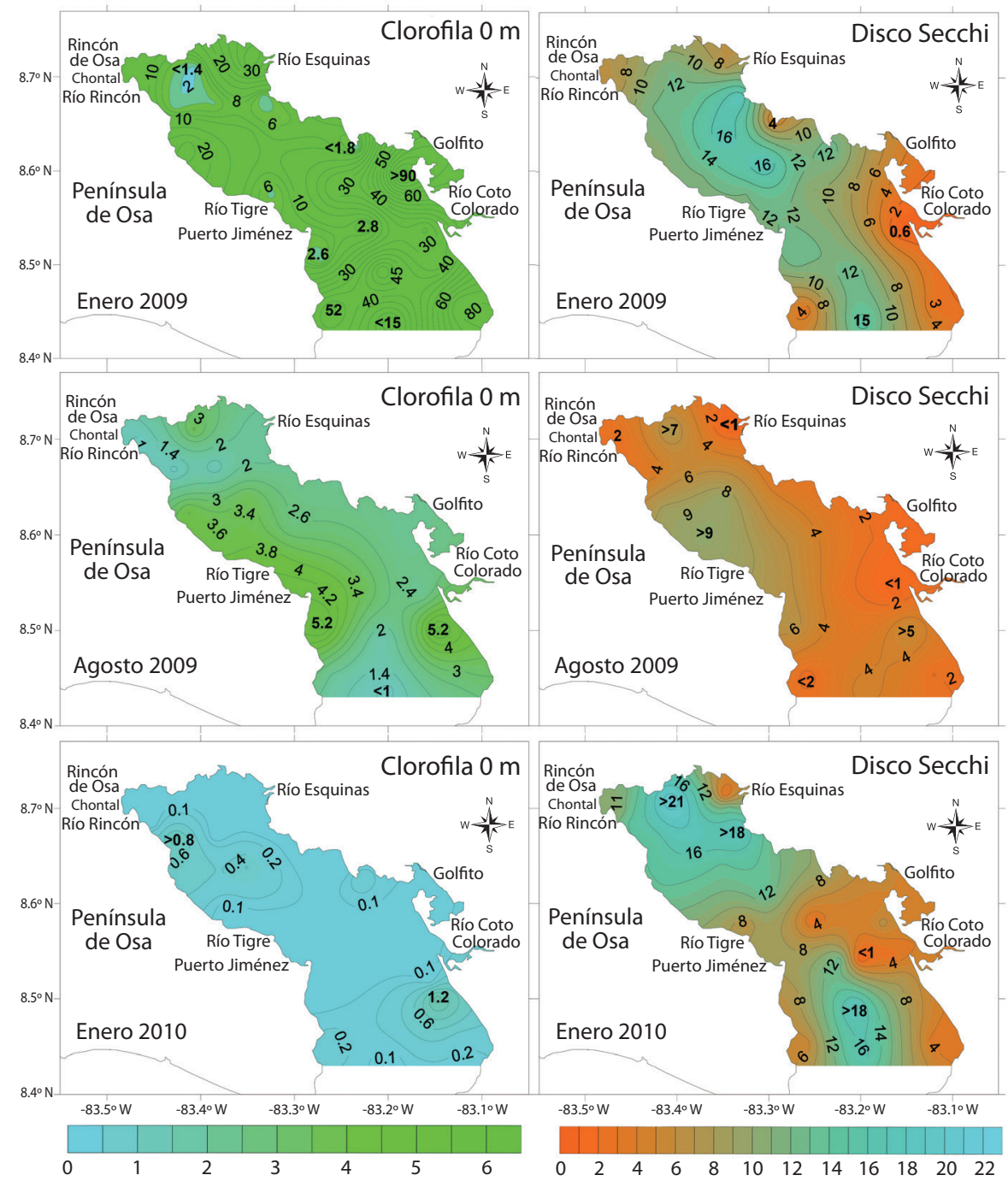

Fig. 9. Clorofila- $a(\mu \mathrm{Mol} / \mathrm{L})$ en superficie (columna izq) y profundidad disco de Secchi (m) (columna der) durante las campañas de enero 2009 (arriba), agosto 2009 (centro) y enero 2010 (abajo).

Fig. 9. Chlorophyll- $a(\mu \mathrm{Mol} / \mathrm{L})$ in surface (left column) and Secchi disk depth (m) (right column) during the survey in January 2009 (top), August 2009 (center) and January 2010 (bottom).

disminuyendo a medida que se aleja de la desembocadura del río. En la estación seca 2009 los valores de sigma-T cerca de la boca del Río Coto Colorado estuvieron apenas por debajo de 12 (Fig. 12), y la isopicna de $18 \mathrm{~kg} / \mathrm{m}^{3}$ no logra alcanzar la estación 17a. En la estación lluviosa, el valor de sigma-T en superficie cerca de la boca del río en la estación 19, disminuye por debajo de $10 \mathrm{~kg} / \mathrm{m}^{3}$, mientras que la isopicna de 18 localizada alrededor de los $5 \mathrm{~m}$ en esa misma estación, alcanza la superficie casi dos kilómetros al oeste de la estación 17.

La Fig. 13 ilustra los valores del número de Richardson (Ri) en la boca del Río Coto Colorado en la estación 19 para agosto 2009. La estimación del número de Richardson (Ri) arrojó indicios de cómo y a qué profundidades se está produciendo mezcla por esfuerzo de 

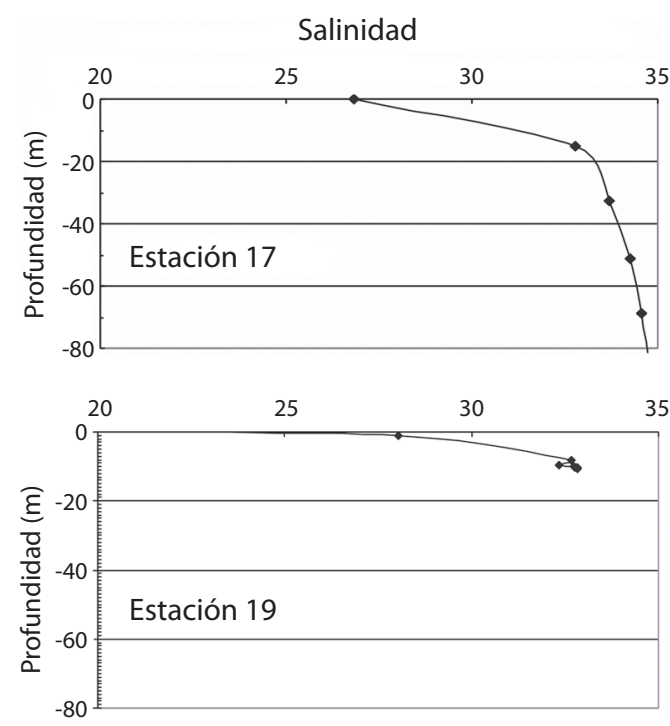

Fig. 10. Perfil de salinidad en las estaciones 17 y 19 durante agosto 2009.

Fig. 10. Salinity profile at the 17 and 19 sample stations during August 2009.

corte (cizallamiento) o se encuentran capas estables. Los valores de Ri en la estación 19 pueden observarse en la Fig. 13, e indican que existe una estabilidad importante en los primeros cinco o seis metros de la columna de agua. Éste patrón cobra más fuerza durante la estación lluviosa, donde la capa superficial, formada por agua salobre (salinidades $>18$, Fig. 11), se muestra estable. Luego entre los 8 y $10 \mathrm{~m}$ de profundidad, comienzan a erosionarse verticalmente las capas de agua y se produce una mezcla importante que se observa tanto en la estación seca como en la lluviosa. Según Mack y Schoeberlein (2004), la misma mezcla puede redistribuir la densidad y el efecto de corte, modificando así los valores de Ri más allá de lo que provocó la mezcla en un principio. A los $11 \mathrm{~m}$ de profundidad muy cerca del fondo, vuelve a mostrarse una capa estable en ambas estaciones del año.

Finalmente y de acuerdo a la profundidad del disco de Secchi, se encontró relación entre la concentración de clorofila- $a$ y la transparencia del agua para los datos correspondientes a la estación lluviosa en 2009, arrojando un $\mathrm{R}^{2}=0.337 \quad(\mathrm{P}=0.05)$ como se muestra en la Fig. 14.

\section{DISCUSIÓN}

Variabilidad hidrográfica en Golfo Dulce (GD): Los cambios de temperatura y salinidad encontrados modificaron la estratificación de la columna de agua, (Sigma-T) a lo largo del transecto central, impactando la mezcla y el intercambio vertical de agua. Solamente en enero 2009 se observa una estructura con una picnoclina definida, localizada entre 12 y $50 \mathrm{~m}$, separando una capa superficial homogénea (Sigma-T $\leq 20 \mathrm{~kg} / \mathrm{m}^{3}$ ) de la capa profunda (Sigma-T $\geq 25 \mathrm{~kg} / \mathrm{m}^{3}$ ). Esto ocurre debido a la fuerza de la picnoclina que inhibe la mezcla. Por otro lado en agosto 2009 y enero 2010 la estratificación de la columna de agua se extendió directamente desde la superficie hasta $60 \mathrm{~m}$ de profundidad, pero la estructura vertical de densidad estuvo determinada en forma diferenciada por la salinidad y la temperatura ya que en agosto del 2009 el alto contenido de agua fresca determinó fuertemente la densidad y estratificación de la capa superior, mientras que en enero 2010, con escaso aporte de agua fresca y alta insolación, la densidad y estratificación estuvieron dominadas por el calentamiento de la capa superior. El efecto preponderante de la estratificación térmica durante ENOS 2010 quedó ilustrado por la variabilidad en la profundidad de la isopicna de $24 \mathrm{~kg} / \mathrm{m}^{3}$, localizada en la base de la capa superior estratificada. El aumento en la estratificación durante una estación seca extrema como la de enero 2010 fue resultado por un lado del aumento en la insolación y al hecho que la temperatura del agua fresca introducida al GD es inferior a la temperatura superficial del mar.

Caracterización física de las plumas en Golfo Dulce: Un perfil característico de salinidad en ambientes estuarinos, según Lentz \& Limeburner (1995), típicamente revela tres distintas regiones: i) una delgada capa cercana a la superficie, formada por agua de baja salinidad 

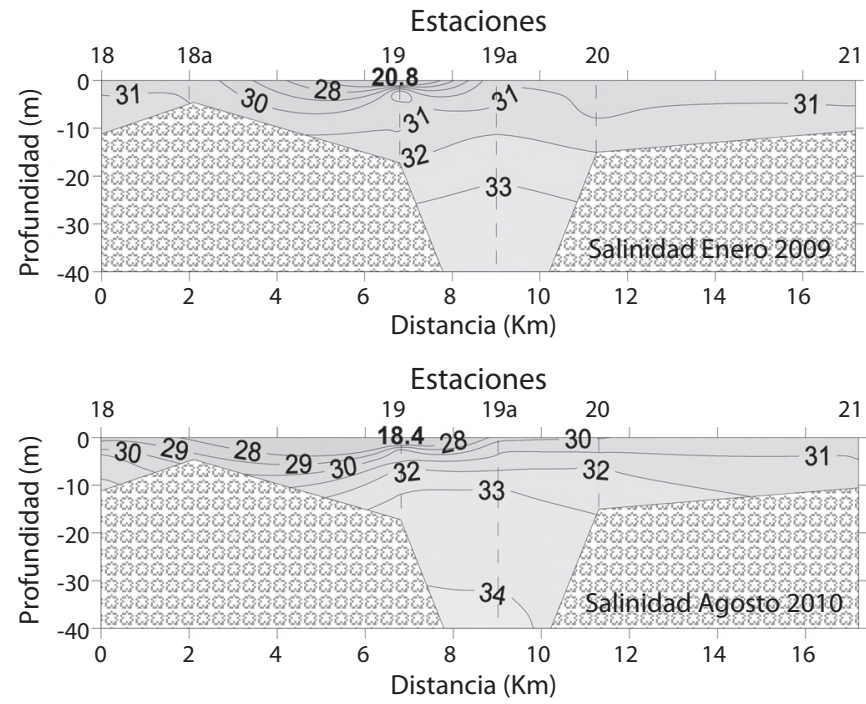

Estaciones

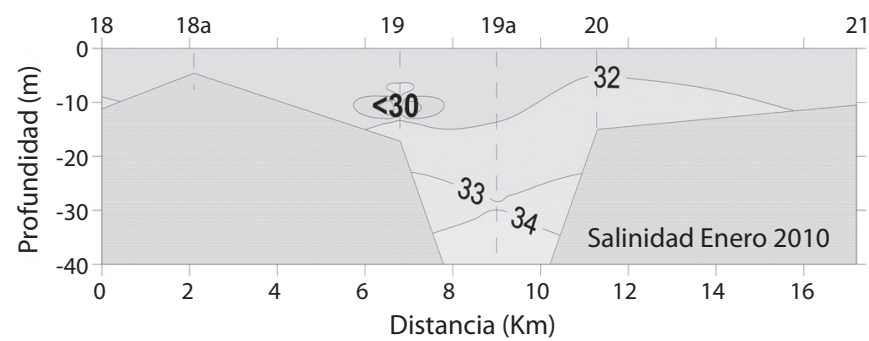

Fig. 11. Distribución vertical de salinidad en el Transecto TC2 durante enero 2009 (arriba), agosto 2009 (centro) y enero 2010 (abajo).

Fig. 11. Salinity vertical distribution in the TC2 Transect during January 2009 (above), August 2009 (center) and January 2010 (bottom).

verticalmente uniforme, ii) debajo de esta capa superior hay un fuerte gradiente de salinidad o haloclina, iii) la capa inferior más salada. En algunos casos, por debajo de los $20 \mathrm{~m}$ aproximadamente, la capa inferior es de agua oceánica con mayor salinidad relativamente constante. El grosor de la pluma de un río se estima como el grosor de la capa superficial más la mitad del grosor de la haloclina.

El modelo del perfil de salinidad en Golfo Dulce muestra una ausencia de haloclina, observándose sólo una estratificación superficial que se hace más evidente en las estaciones cercanas a la desembocadura de los ríos, sumado a que gran parte del resto del golfo es dominado por condiciones más oceánicas aún y cuando toda su superficie mostró una salinidad siempre por debajo de 32. Con nueve perfiles de salinidad analizados el valor promedio del grosor de la pluma del Río Coto Colorado (4.66 $\pm 4.16 \mathrm{~m})$, en particular guarda proporción razonable en relación al volumen de agua que vierte en su desembocadura, unos $177 \mathrm{~m}^{3} / \mathrm{s}$ según Quirós (2003) y Svendsen et al. (2006). El mayor espesor detectado está relacionado con la estación lluviosa, cuando el caudal del río aumenta, sin embargo y aunque agosto fue el mes con mayor precipitación durante el 2009 con $639.6 \mathrm{~mm}$ (IMN, 2009), los días de muestreo 25 y 26 llovió apenas 2.0 y $0.5 \mathrm{~mm}$ respectivamente y dos 

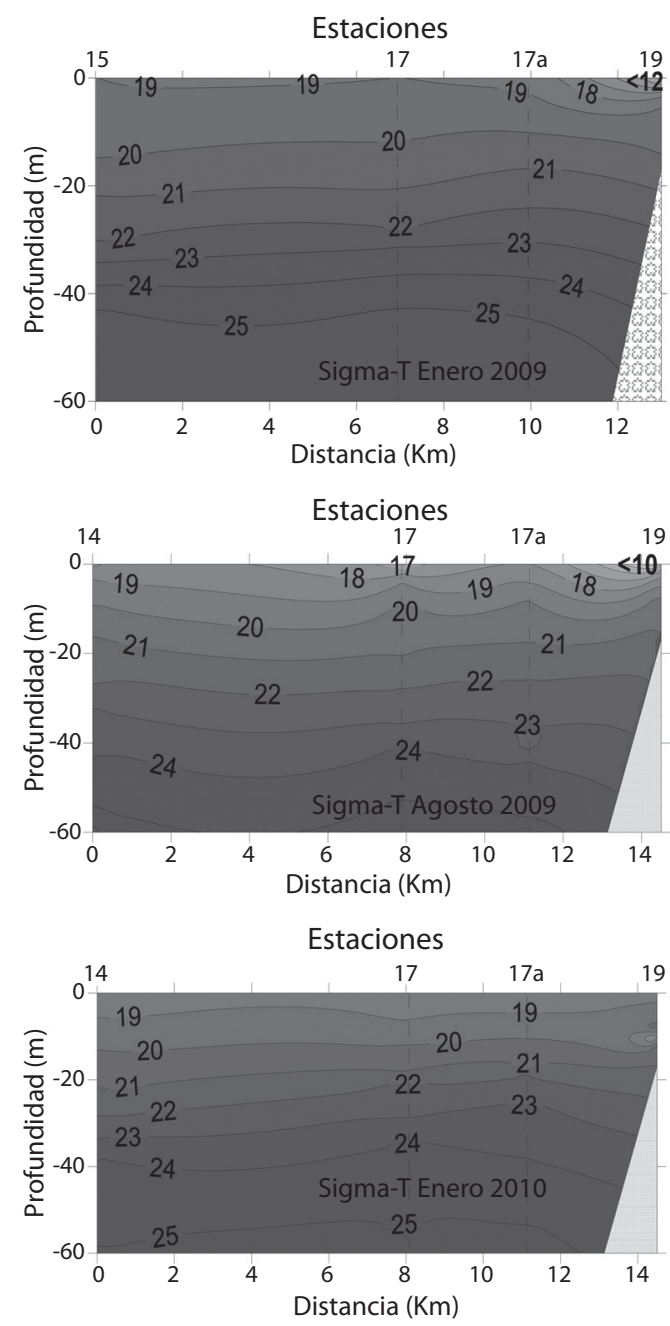

Fig. 12. Distribución vertical de Sigma-T en el Transecto TC1 durante enero 2009 (arriba), agosto 2009 (centro) y enero 2010 (abajo).

Fig. 12. Vertical distribution of Sigma-T in the TC1 Transect during January 2009 (above), August 2009 (center) and January 2010 (bottom).

días después (28/08/2009), se registró el mayor valor del mes $(93.0 \mathrm{~mm})$ nos da una idea de cuánto podría variar su volumen de descarga.

Al notar las diferencias entre un perfil típico de salinidad (Lentz \& Limeburner, 1995) y los perfiles obtenidos, que no muestran una haloclina estrictamente, sino una estratificación superficial, se estimó además el grosor de la pluma del Río Coto Colorado usando como

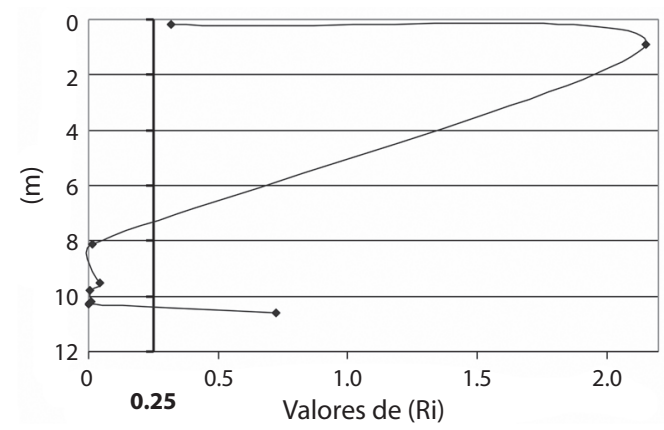

Fig. 13. Valores del número de Richardson (Ri) en la boca del Río Coto Colorado (estación 19), en agosto 2009.

Fig. 13. Richardson number values (Ri) values in the Coto Colorado river mouth (station 19), August 2009.

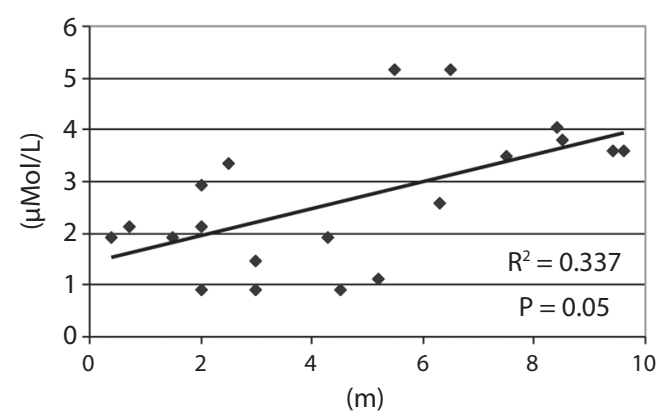

Fig. 14. Correlación entre la lectura del disco Secchi y la concentración de clorofila- $a$ para la estación lluviosa, agosto 2009.

Fig. 14. Correlation between Secchi disc measurement and chlorophyll- $a$ concentration for the rainy season, August 2009.

referencia la isohalina de 30 . La distribución vertical de salinidad a lo largo del transecto TC2 orientado en sentido norte-sur, perpendicular a la boca del Río Coto Colorado, dibuja la presencia de su pluma con valores $\leq 30$ de salinidad, ocupando los primeros $4 \mathrm{~m}$ de la columna de agua entre las estaciones 19 y 18 (Fig. 11), en enero 2009 acentuándose notablemente en agosto. Esto ocurre ya que la pluma del Río Coto Colorado es empujada levemente hacia el norte por acción de las corrientes, desplazando el agua superficial hacia el interior del golfo. En enero 2010, periodo bajo la influencia de ENOS, ya no se observa en superficie rastro alguno de esta pluma. 
La isopicna de $24 \mathrm{~kg} / \mathrm{m}^{3}$ en el transecto TC1 es empujada a una profundidad mayor en la estación lluviosa. En el caso del Río Coto Colorado en Golfo Dulce, la picnoclina es casi una haloclina, mostrándose más fuerte y marcada durante la estación lluviosa.

De acuerdo con Hetland (2004), en una pluma pueden encontrarse dos típicas regiones diferenciadas por los procesos dominantes de mezcla vertical: La primera región o "campo cercano", es un área cercana a la boca del río, donde se experimenta una activa mezcla vertical tanto como se dispersa la descarga del estuario y entra agua profunda a través de la mezcla ocasionada por el esfuerzo de corte (shear). La otra región es un "campo lejano" donde la mezcla inducida por el viento es el mecanismo dominante que propicia la entrada de agua profunda a la pluma. Esto concuerda con Lentz \& Limeburner (1995) que mencionan dos factores que pueden influir en el grosor de la pluma: la mezcla directa por acción del viento y la fuerza de la picnoclina (haloclina) la cual tiende a inhibir la mezcla.

Al no observarse ningún ascenso de las isopicnas, se infiere que la mezcla cerca de la desembocadura de los ríos en Golfo Dulce esta dominada por la acción del viento, como ocurre en ese "campo lejano" descrito por Hetland (2004). De todo esto se deduce que existe estabilidad en la mayoría de las estaciones alrededor de los $40 \mathrm{~m}$ de profundidad (valores $\mathrm{Ri}>0,25)$ debido a que no hay estratificación. De acuerdo con Lei (2002), el Coto Colorado y Esquinas aportan el $90 \%$ del agua dulce que entra al golfo a través de los ríos. Acá queda clara la influencia de estos dos grandes ríos en Golfo Dulce durante la estación lluviosa, que según Quesada-Alpízar y Cortés (2006) se extiende desde abril hasta diciembre y puede llegar superar los $5000 \mathrm{~mm}$ de precipitación anual.

Transparencia y Clorofila- $a$ : $\mathrm{Al}$ igual que en este trabajo Silva y Acuña-González (2006) encontraron concentraciones mayores de este pigmento en la época seca, aunque dentro de la Bahía de Golfito, área cercana a la zona de estudio de este trabajo. Estos valores resultaron especialmente altos, los mayores registrados en este estudio y que posiblemente se deban a una floración o "bloom" extraordinario de fitoplancton, aunque no se tengan mayores pruebas que apoyen esta hipótesis.

Temperaturas mayores a $30.01^{\circ} \mathrm{C}$ tienen un efecto negativo en el crecimiento del fitoplancton y aunque existen otros factores que influyen en el desarrollo de estas comunidades como la disponibilidad de nutrientes, la penetración de luz en el agua, el viento y las corrientes que las dispersan (Lei, 2002), es posible que las bajas concentraciones de clorofila- $a$ registradas durante enero 2010 (Clorofila- $a_{M}=0.46 \pm 0.40 \mathrm{uMol} / \mathrm{L}$ ) fueron causadas por la alta temperatura en la superficie debido a los efectos de ENOS, y probablemente se encuentren concentraciones más altas de este pigmento a profundidad que en superficie.

Es necesario tomar en cuenta la luz y su penetración en el campo subacuático. Según Rincón-Alejos, Astor, Müller-Karger, Verela y Odriorzola (2008) dentro de las plumas estuarinas generalmente hay concentraciones de nutrientes suficientes para el crecimiento microalgal, sin embargo, es restringido el paso de la luz hacia las capas inferiores, inhibiéndose el proceso de fotosíntesis a profundidad y limitando el desarrollo del fitoplancton sólo en las capas más superficiales. En los frentes, justo donde el gradiente es más marcado y se da el cambio abrupto de densidad, el fitoplancton puede desarrollarse mejor ya que existe una combinación de condiciones óptimas de luz y disponibilidad de nutrientes (Rincón-Alejos et al., 2008). Esto explica la ubicación de las máximas concentraciones de clorofila- $a$ en las estaciones 18 y 21 en enero 2009.

\section{CONCLUSIONES}

Los resultados y valores de los parámetros oceanográficos obtenidos en este estudio para Golfo Dulce durante 2009 y 2010, confirman la presencia de una capa superficial cálida y de baja salinidad, influenciada en gran parte por la descarga fluvial a través de ríos y escorrentías, 
que se acentúa durante la estación lluviosa y fortalece la estratificación superficial, debilitándose durante la estación seca, cuando la capa superficial está más mezclada. Por debajo de los 50-60m el agua se encontró más estable durante ambas estaciones del año, es decir, no hubo mayor cambio en los valores. Esto coincide parcialmente con los resultados reportados en la literatura consultada para la zona de estudio (Lei, 2002; Quesada-Alpízar \& MoralesRamírez 2004; Quesada-Alpízar y Cortés 2006; Svendsen et al., 2006).

Existen dos ríos importantes en el flujo de agua dulce hacia el golfo, éstos son el Coto Colorado y el Esquinas. Estos dos ríos con sus descargas disminuyeron considerablemente la salinidad $(<30)$ en un área $>250 \mathrm{~km}^{2}(36.8 \%$ de la superficie total) durante la estación lluviosa, ocupando casi la totalidad del margen este del Golfo Dulce.

Este ha sido un estudio general sobre la hidrografía del golfo y la presencia de aguas continentales en él. Se recomienda realizar un estudio más enfocado en las plumas y sus zonas de influencia, con una densidad mayor de estaciones de muestreo incluyendo aguas arriba de la desembocadura de los ríos y sitios cercanos a los asentamientos humanos. Implementar además un programa de monitoreo ejecutado por instituciones académicas y de investigación, proyectado a mediano y largo plazo, donde se dé seguimiento a la mayor cantidad de variables oceanográficas y ambientales posible para obtener una visión más cercana a la realidad sobre la dinámica de las plumas en Golfo. En caso que logre crearse una categoría de manejo en Golfo Dulce, su plan de manejo debería considerar el área de dispersión de las plumas estuarinas, que en este estudio fue de $250 \mathrm{~km}^{2}$ en un área de $680 \mathrm{~km}^{2}$, lo que equivale al $36.8 \%$ de la superficie total de Golfo Dulce.

\section{AGRADECIMIENTOS}

Profundo agradecimiento al Programa de Becas para Estudios Académicos de Postgrado del Departamento de Desarrollo Humano, Educación y Cultura de la Organización de Estados
Americanos (OEA), ejecutado con asistencia del Latinamerican Schoolarship Program for the America`s Universitys (LASPAU). Al Departamento de Oceanografía Física de la Universidad Nacional (UNA) especialmente a Carlos Delgado y Rebeca Quesada. Al Programa de Maestría en Ciencias Marinas y Costeras (PROCMAR) de la UNA. A Ana Yury Saravia del Laboratorio de Química Marina de la UNA. Al personal de la Estación de Biología Marina de la UNA en Puntarenas. A Yrene Astor, Lenin Oviedo, Flia. Martínez, Taboga, Wagner Quirós, International Student Voluntier (ISV). Al Greg Gund Memorialship Foundation a través de Guido Saborío y Fundación Amigos de OSA.

\section{RESUMEN}

Variables oceanográficas en 33 estaciones en el Golfo Dulce, Costa Rica, entre 2009 y 2010 fueron analizadas a través de perfiles con CTD, donde la salinidad, la temperatura, sigma-T y las corrientes se estudiaron en la columna de agua. Disco Secchi y clorofila- $a$, fueron medidos a nivel superficial.. El objetivo fue estimar el área de dispersión de las plumas estuarinas a través del seguimiento de las variables físicas temperatura, salinidad y sigma-T. En agosto 2009, entre los ríos Coto Colorado y Esquinas disminuyeron la salinidad $(<30)$ en un área de $250 \mathrm{~km}^{2}$ aproximadamente, un $37 \%$ de la superficie total, creándose frentes termohalinos a lo largo de casi todo el golfo. Se documentó el efecto de El Niño Oscilación del Sur en enero 2010 durante su fase de acentuada intensidad. Las concentraciones de clorofila- $a$ se correlacionaron en un $33.7 \%(\mathrm{P}=0.05)$ con la transparencia del agua según el disco Secchi en la estación lluviosa. Se caracterizó el agua superficial del Golfo Dulce por tener salinidad $<31.5$ temperatura $>27^{\circ} \mathrm{C}$ y ocupar los cinco primeros metros de la columna de agua.

Palabras clave: Oceanografía física, descarga de ríos, clorofila- $a$, El Nino Oscilacon del sur.

\section{REFERENCIAS}

Acevedo-Gutiérrez, A., \& Matthew, A. (2005). Association pattern of bottlenose dolphins in Costa Rica: Constant Companions and Casual Acquaintances. San Diego, California: Book of abstract XVI Biennial Conference on the Biology of Marine Mammals.

Acuña-González, J., Vargas-Zamora, J. A., \& CórdobaMuñoz, R. (2006). A snapshot view of some vertical 
distributions of water parameters at a deep (200m) station in the fjord-like Golfo Dulce, embayment, Costa Rica. Revista de Biología Tropical, 54(Suppl. 1), 193-200.

Bonilla, J., Senior W., Bugden, J., Zafiriou, O., \& Jones, R. (1993). Seasonal distribution of nutrient and primary productivity of the eastern continental shelf of Venezuela as influenced by the Orinoco River. Journal of Geophysical Research, 98(C-2), 2245-2257.

Dalsgaard, T., Canfield, D. E., Petersen, J., Thamdrup, B., \& Acuña-González, J. (2003). N2 production by the anammox reaction in the anoxic water column of Golfo Dulce, Costa Rica. Nature, 422(6932), 606-608.

Fairbridge, R. W. (1980). The Estuary: Its definition and geodynamic cycle. In E. Olausson, \& I. Cato (Eds.), Chemistry and Biogeochemistry of Estuaries (pp. 1-35). Chichester: Wiley.

Ferdelman, T. G., Thamdrup, B., Canfield, D. E., Glud, R. N., Kuever, J., Lillebæk, ... Wawer, C. (2006). Biogeochemical controls on the oxygen, nitrogen and sulfur distributions in the water column of Golfo Dulce: an anoxic basin on the Pacific coast of Costa Rica revisited. Revista de Biología Tropical, 54(1), 171-191.

Fiedler, P. C., \& Talley, L. D. (2006). Hydrography of the eastern tropical Pacific: A review. Progress in Oceanography, 69(2-4), 143-180.

Fofonoff, P., \& Millard, R. C. Jr. (1983). Algorithms for computation of fundamental properties of seawater, Unesco 1983. UNESCO Technical Paper in Marine Science, 44, 53.

Hebbeln, D., Beese, D., \& Cortés, J. (1996). Morphology and sediments structure in Golfo Dulce, Costa Rica. Revista de Biología Tropical, 44(3), 1-10.

Hetland, R. D. (2004). Water mass structure of wind forced river plumes. Journal of Physical Oceanography, 35(9), 1667-1688.

Instituto Meteorológico Nacional (IMN). (2009). Datos Climáticos. Costa Rica: Instituto Meteorológico Nacional.

Lei, Z. (2002). A Marine and coastal dynamics and primary production response in Golfo Dulce, Costa Rica. A multi-sensor satellite aproach (Master thesis). International Institute of Geoinformation and Earth Observation, The Netherlands.

Lentz, S., \& Limeburner, R. (1995). The Amazon River plume during AMASSEDS: Spatial characteristics and salinity variability. Journal of Geophysical Research, 100(C-2), 2355-2375.

Mack, S. A., \& Schoeberlein, H. C. (2004). Richardson Number and Ocean Mixing: Towed Chain Observations. Journal of Physical Oceanography, 34, 736-754.
Mann, K., \& Lazier, J. (1996). Dynamics of Marine Ecosystems. Second Edition. Oxfor, UK: Blackwell Science.

Martínez, G., Alvarado, J., \& Senior, W. (2001). Estudio físico-químico de las aguas superficiales de la cuenca baja y pluma del Río Manzanares. Interciencia, 26(8), 342-351.

Mösso, C., Mestres, M., Sierra, J. P., Sánchez-Arcilla, A., González del Río, J., Rodilla, M., ... Cruz, A. (2003). Dinámica de las plumas de dos ríos en las costas del mar Mediterráneo Español. Implicaciones para la gestión costera. Tortosa, España: IV Congreso Iberico de Gestion y Planificacion del Agua.

Oviedo, L. (2008). Análisis del uso de hábitat del delfin manchado pantropical Stenella attenuata (Cetacea: Delphinidae) en el Golfo Dulce, Costa Rica (Tesis de Maestría). Universidad Nacional de Costa Rica, Costa Rica.

Quesada-Alpízar, M., \& Morales-Ramírez, A. (2004). Comportamiento de las masas de agua en el Golfo Dulce, Costa Rica, durante El Niño 97-98). Revista de Biología Tropical, 52(2), 95-103.

Quesada-Alpízar, M., \& Cortés, J. (2006). Los ecosistemas marinos del Pacífico Sur de Costa Rica: estado del conocimiento y perspectivas de manejo. Revista de Biología Tropical, 54, 101-145.

Quirós, G. (2003). Circulación del Golfo Dulce: un fiordo tropical. Tópicos Meteorológicos y Oceanográficos, 10(2), 75-83.

Richards, F. A. (1965). Anoxic basins and fjords. In J. P. Riley, \& G. Skirrow (Eds.), Chemical Oceanography (pp. 611-643). London, England: Academic Press.

Richards, F. A., Anderson, J. J., \& Cline, J. D. (1971). Chemical and physical observations in Golfo Dulce, an anoxic basin of the pacific coast of Costa Rica. Limnology and Oceanography, 16(1), 43-50.

Rincón-Alejos, F., Astor, Y., Müller-Karger, F., Verela, R., \& Odriorzola, A. L. (2008). Características oceanográficas del flujo en Boca de Dragón, Venezuela. Memoria de la Fundación La Salle de Ciencias Naturales, 168, 7-24.

Secretaría de la Convención de Ramsar. (2006). Manual de la Convención de Ramsar: Guía a la Convención sobre los Humedales (Ramsar, Irán, 1971), 4a. edición. Gland, Suiza: RAMSAR.

Sierra, C., Vartanián, D., \& Polimeni, J. (2006). Caracterización social, económica y ambiental del Área de Conservación de Osa 2da edición. Costa Rica: Ministerio del Ambiente y Energía.

Silva, A. M. (2006). Golfo Dulce en peligro. Girasol Digital, 9(32), 38. Ecuperado de http://www.vinv.ucr. ac.cr/girasol/archivo/Girasol32/golfo.htm

Silva A. M. \& Acuña-González, J. (2006). Caracterización físico-química de dos estuarios en la bahía de Golfito, 
Golfo Dulce, Pacífico de Costa Rica. Revista de Biología Tropical, 54(Suppl. 1), 241-256.

Sistema Nacional de Áreas de Conservación (SINAC). (2008). GRUAS II: Propuesta de Ordenamiento Territorial para la conservación de la biodiversidad de Costa Rica. Volumen 3: Análisis de Vacios en la Representatividad e Integridad de la biodiversidad marina y costera. San José, Costa Rica: Ministerio de Ambiente, Energía y Telecomunicaciones.

Spongberg, A. L. (2004). PCB contamination in marine sediments from Golfo Dulce, Pacific coast of Costa Rica. Revista de Biología Tropical, 52, 23-32.
Strickland, J. D. \& Parsons, T. R. (1977). A Practical Handbook of Seawater Analysis. Ottawa, Canada: Bulletin 167 Fisheries Research Board of Canada.

Svendsen, H., Rosland, R., Myking, S., Vargas, J. A., Lizano, O. G., \& Alfaro, E. J. (2006). A physicaloceanographic study of Golfo Dulce, Costa Rica. Revista de Biología Tropical, 54(Suppl. 1), 147-170.

Wolff, M., Hartmann, H. J., \& Koch, V. (1996). A pilot trophic model for Golfo Dulce, a fjord-like tropical embayment, Costa Rica. Revista de Biología Tropical, 44(Suppl. 3), 215-231. 
